Pacific

Journal of

Mathematics

\title{
ON INFINITE UNRAMIFIED EXTENSIONS
}

Christian Maire 


\title{
ON INFINITE UNRAMIFIED EXTENSIONS
}

\author{
Christian Maire
}

\begin{abstract}
In this article we construct number fields $k$ which have a trivial class group, but an infinite unramified extension.
\end{abstract}

\section{Introduction.}

Let $k$ be a number field. A natural question is: Does $k$ admit an infinite unramified extension?

The answer is no, if the root discriminant of $k$ is less than Odlyzko's bounds.

The answer is yes, if $k$ fails the test of Golod-Shafarevic for a prime number $p$. In that case, we know that there exists an infinite unramified $p$-extension $L$ over $k$.

But generally it is fairly difficult to determin whether $k$ admits an infinite unramified extension.

For this problem we introduce the following unramified extensions of $k$ :

i) $k_{\infty}$ is the maximal unramified Galois extension of $k$.

ii) $k^{(1)}$ denotes the Hilbert field of $k$, i.e., the maximal unramified abelian extension of $k$; its Galois group over $k$ is isomorphic to the class group of $k$ via the Artin map. More generally, let $k^{(i)}$ be the Hilbert field of $k^{(i-1)}, i \geq 1$, where $k^{(0)}=k$. Write $k_{H}=\cup k^{(i)} ; k_{H}$ is the Hilbert tower of $k$. We say that the Hilbert tower is finite (or stops) if $\left[k_{H} / k\right]<\infty$, or infinite otherwise.

iii) For a prime number $p, k_{p}$ will be the $p$-Hilbert tower of $k$, that is to say the maximal $p$-extension of $k$ contained in $k_{H}$.

We have the following inclusions:

$$
k \subset k_{p} \subset k_{H} \subset k_{\infty} .
$$

We recall two facts:

1) There exist fields $k$ for which $k_{H}$ is different from $k_{\infty}$. For instance, the field $k=\mathbb{Q}(\sqrt{3.883})$ has a trivial class group, but there exists an unramified $A_{5}$-extension over $k$.

2) Yamamura [7] has shown that for all imaginary quadratic fields $k$ with a discriminant less than 420 (or 729 under GRH), $k_{\infty}=k_{H}$, and $k_{\infty} / k$ is finite (the tower stops at the first, second or third floor). 
Using some refinements of a theorem of Golod-Shafarevic or genus theory, it is possible to obtain sufficient criteria for the existence of an infinite unramified extension of a given number field $k$. However, these results generally imply that the Hilbert tower of the considered field $k$ is infinite.

In Theorem 3.1 we give various examples of fields, such as the biquadratic field $\mathbb{Q}(\sqrt{17601097}, \sqrt{17380678572159893})$, with a trivial class group and an infinite unramified Galois extension: For each field $k$ of Theorem $3.1, k_{H} / k$ is finite and $k_{\infty} / k$ is infinite.

Moreover, we prove that there exists infinitely many quadratic fields with a finite 2-Hilbert tower, together with an infinite unramified extension of degre $2^{\infty}$ (an extension of degre $2^{\infty}$ is an infinite extension which is the compositum of extensions of degree a power of 2 ).

Note that the calculations were performed using PARI [1].

Acknowlegements. The author wishes to thank X. Roblot for providing Example 4.1.2. He also thanks the referee for comments and for Example 3 in Remark 3.4.1.

\section{Preliminaries.}

In this section, we recall four propositions that will be used in the sequel.

Proposition 2.1 (Genus theory and theorem of Golod-Shafarevich, see [6]). Let $K / k$ be a cyclic extension of degree $p$, and $\rho$ the number of places of $k$ which are ramified in this extension. Denote by $E_{K}$ (resp. $\left.E_{k}\right)$ the units group of $K$ (resp. $k)$.

Suppose that

$$
\rho \geq 3+2 \sqrt{d_{p} E_{K}+1}+d_{p} E_{k},
$$

then the $p$-Hilbert tower of $K$ is infinite.

From then on, we shall write $r=\left\lceil 3+2 \sqrt{d_{p} E_{K}+1}+d_{p} E_{k}\right\rceil+\delta$, where $\lceil$.$\rceil is the ceiling function and where \delta=0$ or 1 depending on whether $3+2 \sqrt{d_{p} E_{K}+1}+d_{p} E_{k}$ is an integer or not.

Proposition 2.2 (Kummer theory). Let $k / \mathbb{Q}$ be a cyclic extension of degree $p$ unramified at $p$, and $K / \mathbb{Q}$ an extension of degree $n$. Suppose that for all places $\mathfrak{Q}$ of $K$, the ramification index of $\mathfrak{Q}$ in $K / \mathbb{Q}$ divides the ramification index of $\mathfrak{q}=\mathbb{Q} \cap \mathfrak{Q}$ in $k / \mathbb{Q}$.

Then the extension $K k / k$ is unramified at the finite places. In particular, if $C$ is the Galois closure of $K$, then $C k / k$ is unramified at the finite places.

Note that in this case, a place $\mathfrak{Q}$ of $K$ which is unramified over $\mathbb{Q}$ will ramifiy in $K k / K$ whenever $\mathfrak{Q} \cap \mathbb{Q}$ is ramified in $k / \mathbb{Q}$.

A consequence of the above is the following well-known result (see [4]): 
Proposition 2.3. Let $P$ be an irreducible real polynomial, with squarefree discriminant $D(P)$. Denote by $C$ the Galois closure of $P$. Then $C / \mathbb{Q}(\sqrt{\operatorname{Disc}(P)})$ is unramified.

Proposition 2.4 (Class group of biquadratic fields). Let $k_{1}=\mathbb{Q}\left(\sqrt{d_{1}}\right)$ and $k_{2}=\mathbb{Q}\left(\sqrt{d_{2}}\right)$ be two disctincts quadratic fields. Let $k_{3}=\mathbb{Q}\left(\sqrt{d_{1} \cdot d_{2}}\right)$ and $F=k_{1} k_{2}$. Assume that:

1) $F / k_{3}$ is uramified at all places;

2) The class groups of $k_{1}$ and $k_{2}$ are trivial;

3) The class group of $k_{3}$ is cyclic of order 2 .

Then the Hilbert tower of $k_{3}$ stops at $F$; in particular, the class group of $F$ is trivial.

Proof. It is known that for $p \neq 2$ (see [5])

$$
\left|c l_{F}(p)\right|=\left|c l_{k_{1}}(p)\right|\left|c l_{k_{2}}(p)\right|\left|c l_{k_{3}}(p)\right|=1,
$$

where $c l_{M}(p)$ denotes the $p$-primary component of the class group of a number field $M$.

Moreover, the 2 -Hilbert tower of $k_{3}$ is exactly $F$ because $c l_{k_{3}}(2)$ is cyclic of order 2. In addition $\left|c l_{F}(2)\right|=1$, and $\left|c l_{F}\right|=1$.

\section{Main result.}

We propose to construct quadratic fields with a finite Hilbert tower, such that they have an infinite unramified extension. Note that in all our examples the tower will stop at the first floor. This point will be proved with the help of Proposition 2.4.

3.1. Construction of infinite unramified extension. We consider the following situation:

1) $K$ is a totally real field of degree $n$ over $\mathbb{Q}$ such that:

i) The discriminant of $K$ is equal to a prime number $l$. Hence the Galois group of the closure of $K$ is $S_{n}$ (see [4]). Let $P$ be a defining polynomial of $K$.

ii) There exist $n-2$ unramified places above $l$ in $K / \mathbb{Q}$.

2) $q_{1}$ and $q_{2}$ are two primes different from $l$ such that:

i) $q_{1} \equiv q_{2} \equiv 3(4)$

ii) $q_{1}$ splits completely in $K$,

iii) there exist $n-1$ places above $q_{2}$ in $K / \mathbb{Q}$.

3) We write $k=\mathbb{Q}\left(\sqrt{l \cdot q_{1} \cdot q_{2}}\right)$, and $M=K k$.

Using Proposition 2.2, we have that all places above $q_{1}$ and $q_{2}$ plus $n-2$ places above $l$ are ramified in $M / K$. Therefore, in the notation of Proposition 2.1 , in the extension $M / K$ we have

$$
\rho=n+(n-1)+(n-2) .
$$


Applying Proposition 2.1 to $M / K$, we conclude that $M$ has an infinite 2-Hilbert tower, denoted by $M_{2}$, if $n$ is at least 7 .

We have from Proposition 2.2 that the extension $M / k$ is everywhere unramified. The above facts imply the following inclusions:

$$
k=\mathbb{Q}\left(\sqrt{l \cdot q_{1} \cdot q_{2}}\right) \subset M \subset M_{2},
$$

where $M_{2} / k$ is an infinite unramified extension. Taking the Galois closure of $M_{2}$ over $k$, we obtain an infinite unramified Galois extension $L$ over $k$. In particular, $k_{\infty} / k$ is infinite.

3.2. The finiteness of $k_{H} / k$. Suppose that we have found primes $l, q_{1}$, $q_{2}$ and a field $K$ of degree $n$ satisfying all the hypotheses of paragraph 3.1. Let $k_{1}, k_{2}$, and $k=k_{3}$ be the quadratic fields of discriminant $l, l . q_{1}$, and $l . q_{1} . q_{2}$, respectively, and put $F=k_{1} k_{2}$. Since $l \equiv q_{1} q_{2} \equiv 1(\bmod 4), F / k_{3}$ is unramified. If, in addition, $k_{1}, k_{2}$ have trivial class groups and $k_{3}$ has class number 2, then the Hilbert tower stops at $F$.

We remark that $c l_{F}$ is trivial, and that the extension $L F / F$ is an infinite unramified Galois extension, because $F / k$ is unramified.

3.3. Finding a good polynomial. We want to find a polynomial of degree 7 verifying condition $1 . i$ and $1 . i i$ of paragraph 3.1. Such a polynomial does not exist in the PARI tables. Consequently, we use the following method:

Pick 7 reals around 0 . Construct the monic polynomial $P^{\prime}$ of degree 7 whose roots are the considered reals, and take $P \in \mathbb{Z}[X]$ the nearest polynomial to $P^{\prime}$. Then by choosing many families of 7 reals, we finally hope to find a good polynomial, i.e., such that:

i) $\operatorname{Disc}(P)=l$;

ii) $P \equiv\left(X-\alpha_{1}\right)^{2} \prod_{i=2,6}\left(X-\alpha_{i}\right)(\bmod l)$.

Indeed, we rapidly obtain the polynomial

$$
P(X)=X^{7}-3 X^{6}-13 X^{5}+28 X^{4}+42 X^{3}-47 X^{2}-31 X+12,
$$

with

$$
l=\operatorname{Disc}(P)=17380678572159893 .
$$

Conditions $1 . i$ and $1 . i i$ of paragraph 3.1 are verified.

3.4. Examples. We find one potential prime $q_{1}$ less than $300000\left(q_{1}=\right.$ $16747)$, and 17 potential primes $q_{2}$ less than 100000 .

Finally, eight of these primes $q_{2}$ are such that $\left(l, q_{1}=16747, q_{2}\right)$ satisfies the conditions of Proposition 2.4 (or paragraph 3.2).

Hence we have:

Theorem 3.1. For the quadratic fields $\mathbb{Q}\left(\sqrt{17380678572159893.16747 . q_{2}}\right)$, with $q_{2}=1051,11863,24659,31583,74527,77339,86579,93491$, one has:

i) $k_{H} / k$ is finite;

ii) $k_{\infty} / k$ is infinite. 


\subsubsection{Remarks.}

1) If we choose $q_{2}$ such that $P$ is totally decomposed modulo $q_{2}$, then $q_{2}$ is totally decomposed in the Galois closure of $P$, and so $q_{2}$ is decomposed in the extension $\mathbb{Q}(\sqrt{l}) / \mathbb{Q}$. Consequently the class group of $\mathbb{Q}\left(\sqrt{l \cdot q_{1} \cdot q_{2}}\right)$ contains the cyclic group (4) [2]. In particular, the Hilbert field of this field is not a biquadratic field so it would be more difficult to control its class group.

2) For each field of Theorem 3.1, there exists an unramified infinite extension $L$ over $F$ with Galois group $G$ such that we have the extension sequence:

$$
1 \longrightarrow H \longrightarrow G \longrightarrow A_{7} \longrightarrow 1,
$$

where $H$ is a non-analytic pro-2-group [3]. Note that $G=[G, G]$.

As a result, the 2-rank of the class group of any nested sequence of extensions $M$ of $F$ contained in $L$ tends to infinity (see [3]). One shall bear in mind that $c l_{F}=(1)$.

3) It would be interesting to find the field of smallest discriminant satisfying the hypotheses of Theorem 3.1. The referee gives the following example:

$$
P(X)=X^{7}+9 X^{6}+13 X^{5}-57 X^{4}-86 X^{3}+120 X^{2}-1,
$$

with $q_{1}=20411$ and $q_{2}=787$.

This example has the desired properties and the biquadratic field $F$ has slighty smaller discrimant than the field in the introduction.

\section{A remark.}

One may try to apply Proposition 2.3 in order to find fields satisfying the conditions of Theorem 3.1.

More precisely, let $P$ be an irreducible totally real polynomial with degree $n$ such that the discriminant of $P$ is equal to $l_{1} \cdot l_{2} \cdot l_{3}$ with $l_{1} \equiv 1(4)$, and $l_{2} \equiv l_{3} \equiv 3(4)$, where $l_{1}, l_{2}, l_{3}$ are primes.

Let $K=\mathbb{Q}(\theta)$, where $\theta$ is a root of $P, k=\mathbb{Q}\left(\sqrt{l_{1} \cdot l_{2} \cdot l_{3}}\right)$, and $M=$ $K k$. Assume that $l_{1}, l_{2}$ and $l_{3}$ are sufficiently decomposed in $K / \mathbb{Q}$. Then for larger $n$, using Propositions 2.1 and 2.3 we obtain that the field $k=$ $\mathbb{Q}\left(\sqrt{l_{1} \cdot l_{2} \cdot l_{3}}\right)$ has an infinite unramified Galois extension.

To show that the Hilbert tower of this field stops we can use an argument similar to the one involved in the previous section.

Yet, the problem becomes: How to find a polynomial satisfying the hypotheses above.

4.1. A method. Let $l_{1} \equiv 1(4)$ and $l_{2} \equiv 3(4)$ be two primes, and put

$$
P(X)=X^{2} \prod_{i=1}^{n-2}\left(X-\alpha_{i}\right)_{-}^{+} l_{1} . l_{2},
$$

where $\alpha_{i}$ are different integers. Changing $\alpha_{i}, l_{1}$, and $l_{2}$, we hope to find some polynomials $P$ such that: 
1) $P$ is irreducible;

2) $P$ is totally real;

3) $\operatorname{Disc}(P)=l_{1} \cdot l_{2} \cdot l_{3}$, for some prime $l_{3}$;

4) $P$ is sufficiently decomposed modulo $l_{3}$;

5) For $i=2$ or $3,\left(\frac{l_{1}}{l_{i}}\right)=-1$.

Note that the last condition is necessary to get $c l_{k_{3}}=(2)[\mathbf{2}]$.

4.1.1. Case $n=17 ; r=32$.

For the definition of $r$, see the paragraph of following Proposition 2.1. In that case, we should have at least two places above $l_{3}$.

We have produced one polynomial:

$$
\begin{aligned}
P(X)= & X^{17}-8 X^{16}-140 X^{15}+1120 X^{14}+7472 X^{13}-59696 X^{12} \\
& -191620 X^{11}+1532960 X^{10}+2475473 X^{9}-19803784 X^{8} \\
& -15291640 X^{7}+122333120 X^{6}+38402064 X^{5} \\
& -307216512 X^{4}-25401600 X^{3}+203212800 X^{2}-4819
\end{aligned}
$$

with $\operatorname{Disc}(P)=l_{1} \cdot l_{2} \cdot l_{3}$, where $l_{1}=61, l_{2}=79$ and $l_{3}=66382900552793321010851526783904690431649057036670997212644$ 289798588293426436298810789013190435989241744842371595711118731 34668406985800955450434299964685089696459.

Note that $\log \left(l_{3}\right) / \log (10) \approx 163$, and that

$$
\begin{aligned}
P(X)= & X^{2}(X-1)(X-2)(X-3)(X-4)(X-5)(X-6) \\
& \cdot(X-7)(X-8)(X+1)(X+2)(X+3)(X+4)(X+5) \\
& \cdot(X+6)(X+7)-61.79
\end{aligned}
$$

There are 5 places above $l_{3}$ which are ramified in $K / k$. So $\rho=15+15+5=$ $35>r$.

4.1.2. Case $n=11 ; r=24$.

$\mathrm{X}$. Roblot comes up with the polynomial:

$P(X)=X^{11}-45 X^{10}+870 X^{9}-9450 X^{8}+63273 X^{7}-269325 X^{6}+$ $723680 X^{5}-1172700 X^{4}+1026576 X^{3}-362880 X^{2}+2483$

with $\operatorname{Disc}(P)=l_{1} \cdot l_{2} \cdot l_{3}$, where $l_{1}=13, l_{2}=191$ and

$l_{3}=1975697671490152075520432855935517362161188018903$.

Here $\rho=9+9+7=25>r$.

4.1.3. Case $n=9 ; r=21$.

We found three polynomials ; for all these cases $\rho=7+7+7=21 \geq r$. 
- $X^{9}-16 X^{8}-122 X^{7}+1100 X^{6}+3709 X^{5}-20524 X^{4}-28068 X^{3}+$ $95760 X^{2}-18467$

with $l_{1}=313, l_{2}=59$, and

$l_{3}=26255115680330741686041335538501141687236954384684227$.

- $X^{9}+35 X^{8}+51 X^{7}-1535 X^{6}-2476 X^{5}+18660 X^{4}+17424 X^{3}-$ $69120 X^{2}+13067$

with $l_{1}=73, l_{2}=179$, and

$l_{3}=7154637362578369142642781505295690026075933100681339$.

- $X^{9}+41 X^{8}+69 X^{7}-1805 X^{6}-3046 X^{5}+22044 X^{4}+21096 X^{3}$ $82080 X^{2}+18511$

with $l_{1}=173, l_{2}=107$, and

$l_{3}=95384503977834605133365739257008489233297152056661447$.

The problem is to calculate the class group of the fields $\mathbb{Q}\left(\sqrt{l_{1}}\right)$ and $\mathbb{Q}\left(\sqrt{l_{1} \cdot l_{2} \cdot l_{3}}\right)$.

\section{Infinite non Galois unramified extension.}

In this section, we prove:

Theorem 5.1. There exists infinitely many quadratic fields (imaginary and real) with a finite 2-Hilbert tower, but with an infinite unramified extension of degre $2^{\infty}$.

Proof (only for the real case) and examples.

Let $K$ be a totally real extension over $\mathbb{Q}$ of degree 8 , such that Disc $(K)=l$ where $l$ is prime number. Let $q_{1}$ and $q_{2}$ be two primes, satisfying:

1) $q_{1} \equiv q_{2} \equiv 3(4)$

2) $q_{1}$ and $q_{2}$ are totally decomposed in $K / \mathbb{Q}$.

Put $N=K \mathbb{Q}(\sqrt{l})$.

Then $q_{1}$ and $q_{2}$ are totally decomposed in the Galois closure of $P$, in particular in $N / \mathbb{Q}$. This extension is of degree 16 .

Using Proposition 2.1 applied to the extension $N\left(\sqrt{q_{1} \cdot q_{2}}\right) / N$, we conclude that $E=N\left(\sqrt{q_{1} \cdot q_{2}}\right)$ has an infinite 2-Hilbert tower, noted $E_{2}$.

Thus, we have the following inclusions:

$$
\mathbb{Q}\left(\sqrt{l \cdot q_{1} \cdot q_{2}}\right) \subset \mathbb{Q}\left(\sqrt{l}, \sqrt{q_{1} \cdot q_{2}}\right) \subset E \subset E_{2},
$$

where all extensions are unramified 2-extensions. Accordingly, $E_{2} / \mathbb{Q}\left(\sqrt{l \cdot q_{1} \cdot q_{2}}\right)$ is an unramified extension of degree $2^{\infty}$.

Moreover, the choice of $q_{1}$ and $q_{2}$ implies that the 2-part of class group of $\mathbb{Q}\left(\sqrt{l \cdot q_{1} \cdot q_{2}}\right)$ is cyclic. As consequence the 2-Hilbert tower stops at the first floor.

If $K / \mathbb{Q}$ exists, Cebotarev's density criterion asserts that there exist infinitely many such fields $\mathbb{Q}\left(\sqrt{l \cdot q_{1} \cdot q_{2}}\right)$. 
For the imaginary quadratic case, one may start with the same $K$ and $q_{1}$ as above and take $q_{2}=-1$. Then $\mathbb{Q}\left(\sqrt{l \cdot q_{1} \cdot q_{2}}\right)$ is an imaginary quadratic field with the desired properties.

5.1. Examples. Consider the following polynomial, found in the PARI tables (E-mail: megrez.math.u-bordeaux.fr, directory pub/numberfields):

$$
P(X)=X^{8}-X^{7}-7 X^{6}+5 X^{5}+14 X^{4}-6 X^{3}-9 X^{2}+X+1,
$$

where

$$
\operatorname{Disc}(P)=1318279381 \text {. }
$$

Then the fields

$$
\mathbb{Q}(\sqrt{1302839.4503991 .1318279381})
$$

and

$$
\mathbb{Q}(\sqrt{-643.1318279381})
$$

satisfy the conditions of Theorem 5.1.

\section{References}

[1] C. Batut, K. Belabas, D. Bernardi, H. Cohen and M. Olivier, User's guide to PARI$G P, 1997$.

[2] G. Gras, Sur les classes d'idéaux dans les extensions cycliques relatives de degré premier l, Ann. Inst. Fourier, 23(4) (1973), 1-48.

[3] F. Hajir, On the growth of p-class groups in p-class field towers, J. Algebra, 188 (1997), 256-271.

[4] T. Kondo, Algebraic number fields with the discriminant equal to that of a quadratic number field, J. Math. Soc. Japan, 42 (1995), 31-36.

[5] T. Kubota, Über den Bizyklischen Biquadratischen Zahlkörper, Nagoya Math. J., 10 (1955), 65-85.

[6] J. Martinet, Tours de corps de classes et estimations de discriminants, Invent. Math., 44 (1978), 65-73.

[7] K. Yamamura, Maximal unramified extensions of imaginary quadratic number fields of small conductors, preprint, 1997.

Received April 15, 1998 and revised June 2, 1998.

LABOratoire A2X - Université Bordeaux I

351, Cours De la Libération

33405 Talence CedeX

FRANCE

E-mail address: maire@math.u-bordeaux.fr 